\title{
Análise temporal da precipitação no aeroporto de São José dos Campos - SP entre os anos de 1982 a 2008 utilizando teste não-paramétrico de aleatoriedade.
}

\author{
Roberto Tadeu de Araujo ${ }^{1}$ \\ Marcela de Ávila Villaron ${ }^{1}$ \\ Marcos Luiz de Andrade Pinto ${ }^{1}$ \\ Cleber Souza Corrêa ${ }^{1}$ \\ ${ }^{1}$ Instituto de Controle do Espaço Aéreo ICEA - Divisão de Pesquisa \\ Pça Mal Eduardo Gomes, 50 Vila das Acácias 1228-903 \\ São José dos Campos - SP, Brasil \\ estudosclimatologicos@icea.gov.br
}

\begin{abstract}
This work tried to search the precipitation series tendency of the Airport of São José dos Campos, using a nonparametric of random statistic test (RUNS TEST), and analyzing the linear series tendency. Monthly precipitation data, total and frequency, were used. These data were collected from the Climatologic Data Bank of ICEA. From time series of real precipitation total, it was used through a filter, a new series, minimized seasonal variability effects. As a result it was observed a declination in the series such linear analyzes as in the nonrandom test Runs.
\end{abstract}

Palavras-chave: precipitation, time series, Runs Test, precipitação, séries temporais, teste de "Runs"

\section{INTRODUÇÃO}

A água é um bem insubstituível à manutenção da vida e a sua circulação na Terra ocorre sob um processo natural conhecido como ciclo hidrológico. Assim, as precipitações pluviométricas representam um papel importante de elo entre os fenômenos atmosféricos e a fase terrestre deste ciclo. Então o conhecimento quantitativo da sua variabilidade espacial sobre as bacias hidrográficas deve ser entendido como imprescindível ao eficiente planejamento e gerenciamento dos recursos hídricos. (Paula,2008; Salgueiro; Montenegro, 2008; Santos; Galvíncio; Moura, 2008)

Dentro deste contexto, uma possível redução no volume de chuvas pode ter consequências sociais e econômicas, provocada pela queda das vazões dos rios (Marengo; Alves, 2005).

Marengo e Alves (2005) consideram como uma das possíveis causas da redução sistemática nas vazões e cotas do Rio Paraíba do Sul, as mudanças do regime e de distribuição da precipitação na bacia decorrentes de possíveis mudanças climáticas regionais. Entretanto, em seu estudo concluem que, embora haja uma tendência negativa na vazão nos últimos 50 anos, esta não parece estar associada às variações de chuva na bacia, e sugere que esta diminuição está atrelada a influência antrópica.

É válido ressaltar que em alguns estudos, as séries temporais de precipitação não são suficientemente longas ou reais, isto é, efetivamente coletadas, para detectar variações climáticas.

Araujo et al. (2008) aplicou o mesmo teste não-paramétrico de aleatoriedade em séries mensais de precipitação e nevoeiro para os Aeroportos de Congonhas e Porto Alegre. Os resultados demonstraram que a precipitação e o nevoeiro apresentaram pequenas diminuições ao longo do período analisado (1975-2005), embora não sejam mudanças significativas.

Assim, a fim de complementar o trabalho anterior, este estudo aplicou o teste nãoparamétrico de aleatoriedade nas séries mensais de precipitação, do período de 1982 até 2006, para o Aeroporto de São José dos Campos. Acrescida da análise linear, o objetivo deste 
trabalho foi verificar se a estação está apresentando alguma tendência de diminuição na quantidade mensal de precipitação.

\section{MATERIAIS E MÉTODOS}

\section{1. Área de estudo}

A área de estudo compreende o aeroporto Professor Urbano Ernesto Stumpf, no município de São José dos Campos - SP localizado nas coordenadas 2313'44'S e 045'52'16' 'W, em uma altitude de 646 m (COMAER, 2005).

Está inserida na Depressão do Rio Paraíba do Sul, pertencente aos Cinturões Móveis Neoproterozóicos Sudeste-Sul (IBGE, 2006). Esta área apresenta em uma parcela a vegetação do tipo Cerrado e, em outra, Floresta Ombrófila Densa na sua forma secundária, ambos os tipos com atividade agrária (IBGE, 2004). O clima para a região, segundo Köppen, é do tipo Cwa, com verão quente e inverno seco.

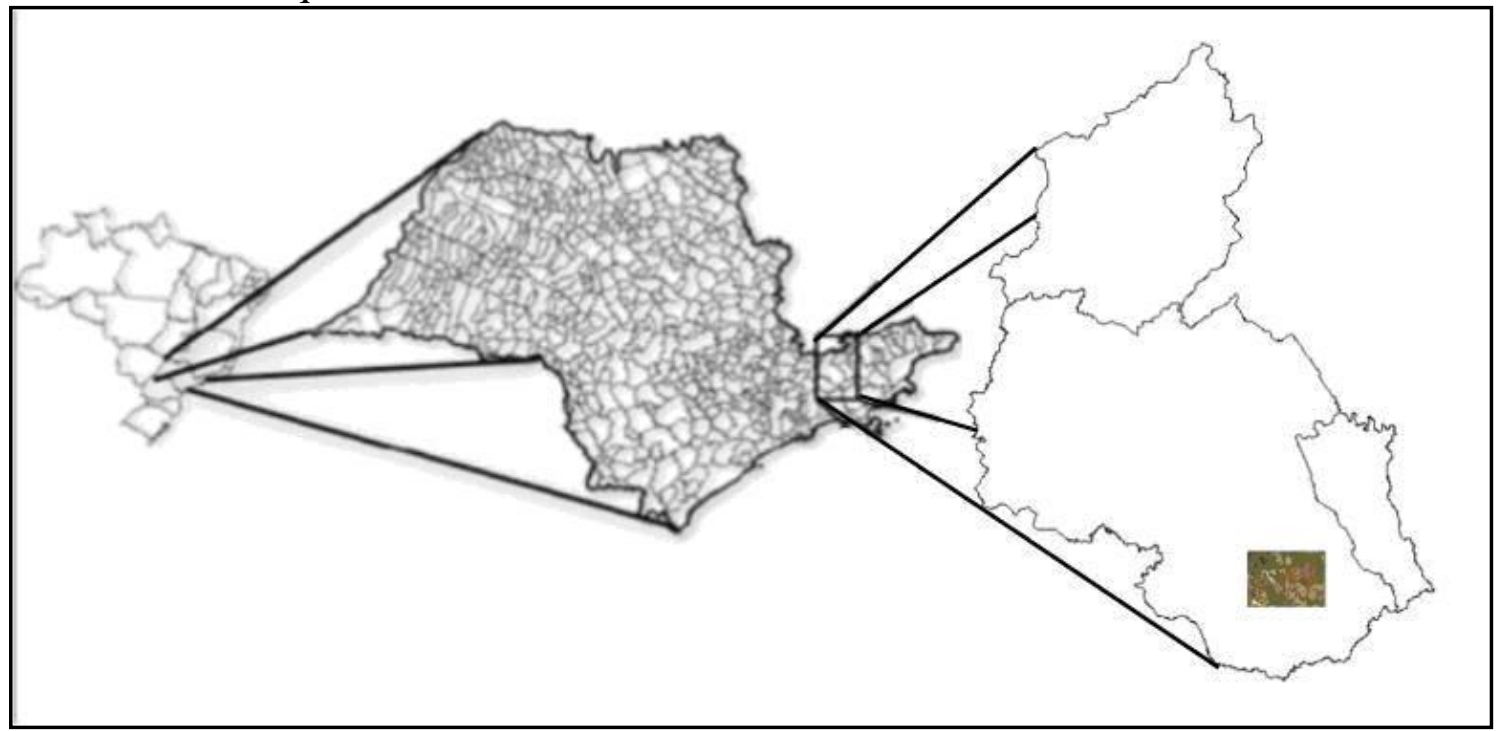

Figura 1 - São José do Campos (SP), Brasil. Fonte: Villaron, 2008.

\subsection{Metodologia}

Para este trabalho foram utilizados dados de precipitação registrados na Estação Meteorológica de Superfície do Aeroporto de São José dos Campos (SBSJ) no período de 1982 a 2006. Os dados estão arquivados no Instituto de Controle do Espaço Aéreo (ICEA), em São José dos Campos - SP. O horário de funcionamento da EMS foi da $0 \mathrm{~h}$ às $23 \mathrm{~h}$ local, sendo realizada uma observação meteorológica a cada hora.

Os testes estatísticos foram aplicados nas séries temporais com informações de total de horas de precipitação e total pluviométrico por um período de 25 anos, com dados mensais.

Além disso, elaborou-se uma nova série, chamada de "corrigida", onde se procurou atenuar os efeitos da sazonalidade, através das equações:

$\mathrm{FS}=\mathrm{A} / \mathrm{B}$

onde FS é igual ao fator sazonal, A é igual a média mensal, e B igual a média de todos os meses e de todo o período.

\section{$\mathrm{C}=\mathrm{D} / \mathrm{FS}$;}

onde $\mathrm{C}$ é igual a nova média mensal corrigida; $\mathrm{D}$ é igual a média mensal real de cada mês e de cada ano; e FS igual ao fator sazonal.

Em seguida, verificou-se a tendência linear nas três séries e foi aplicado o teste nãoparamétrico de aleatoriedade (Teste "RUNS") descrito por Siegel e Castellan (1988), Sheskin 
(2000) e Jianqing e Yao (2003). Este teste observa na série em estudo a mudança de sinal acima e abaixo do valor da média. Em uma série comportada esperar-se-iam valores proporcionais acima e abaixo deste valor. Com aplicação do teste observa-se tendência crescente ou decrescente.

Foi utilizado o software Minitab 15 para gerar as análises.

\section{RESULTADOS E DISCUSSÕES}

Os resultados das análises procuraram investigar as tendências nas séries mensais, desde janeiro de 1982 a dezembro de 2006.

Num primeiro momento, as séries temporais foram apresentadas com análise de tendência linear, cujos valores correspondem à linha retilínea que se ajusta aos dados. Os resultados indicam leve declínio tanto no total de precipitação mensal real (Figura 2), acumulando no $300^{\circ}$ mês 17,0 mm a menos, quanto na frequência do fenômeno (Figura 3), reduzindo a média da quantidade de registros mensais de 63 para 44 .

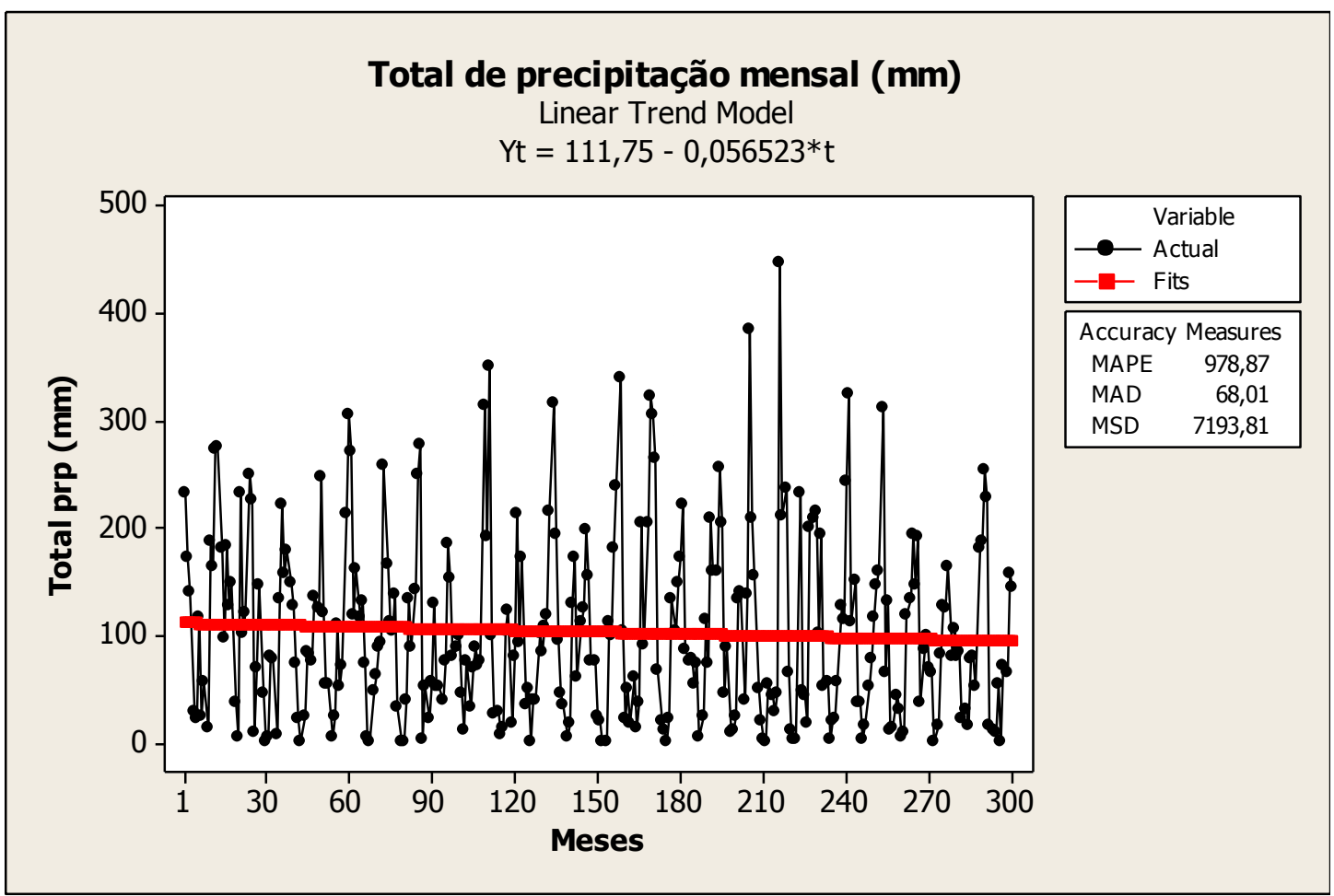

Figura 2 - Total de precipitação mensal com a série real do aeroporto de São José dos Campos entre os anos de 1982 a 2006.

Fonte: ICEA. 


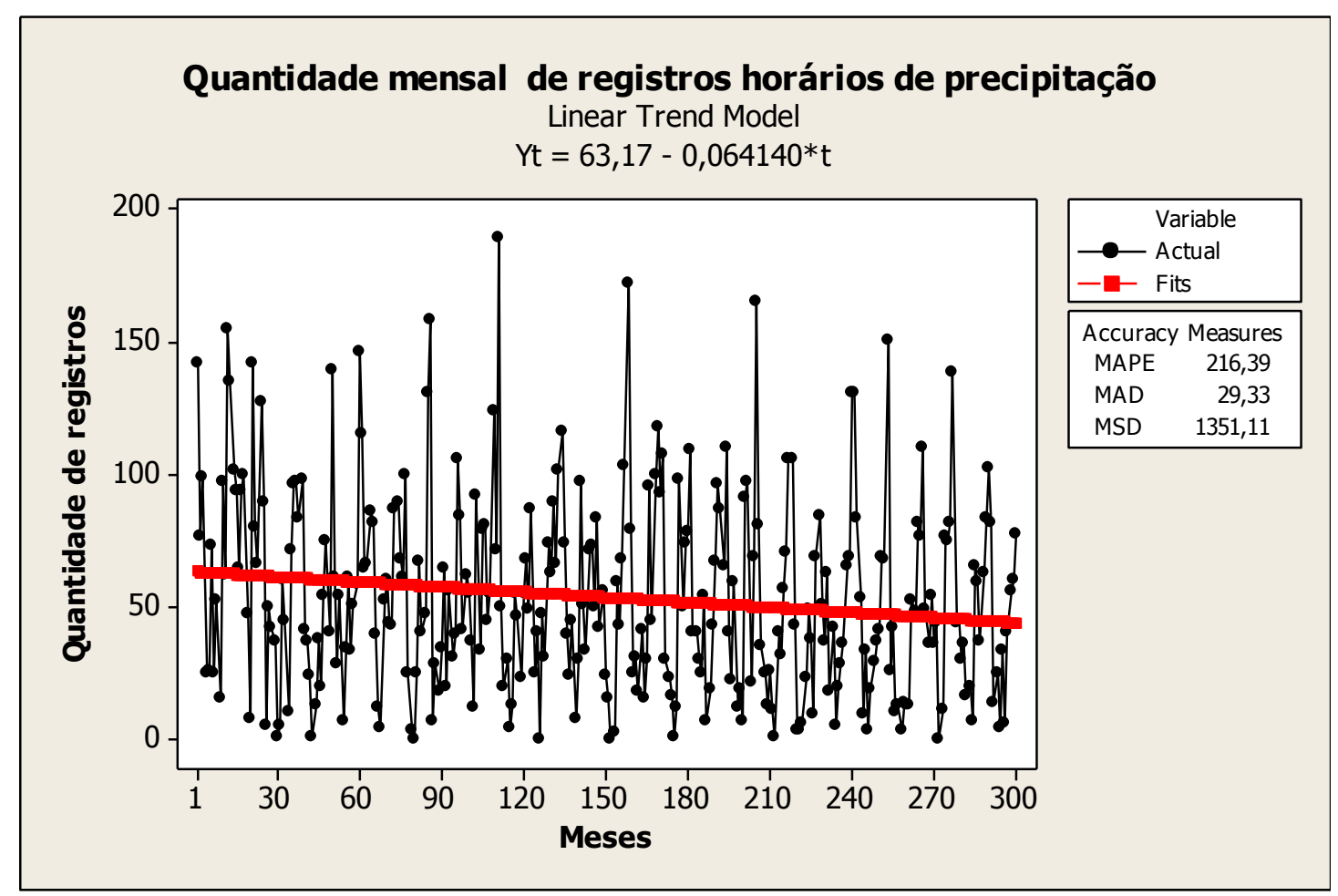

Figura 3 - Quantidade mensal de registros horários de precipitação do aeroporto de São José dos Campos entre os anos de 1982 a 2006.

Fonte: ICEA.

A tendência também se confirmou na série corrigida do efeito sazonal, inclusive com maior relevância, apontando para um decréscimo de $32,9 \mathrm{~mm}$ no final do período.

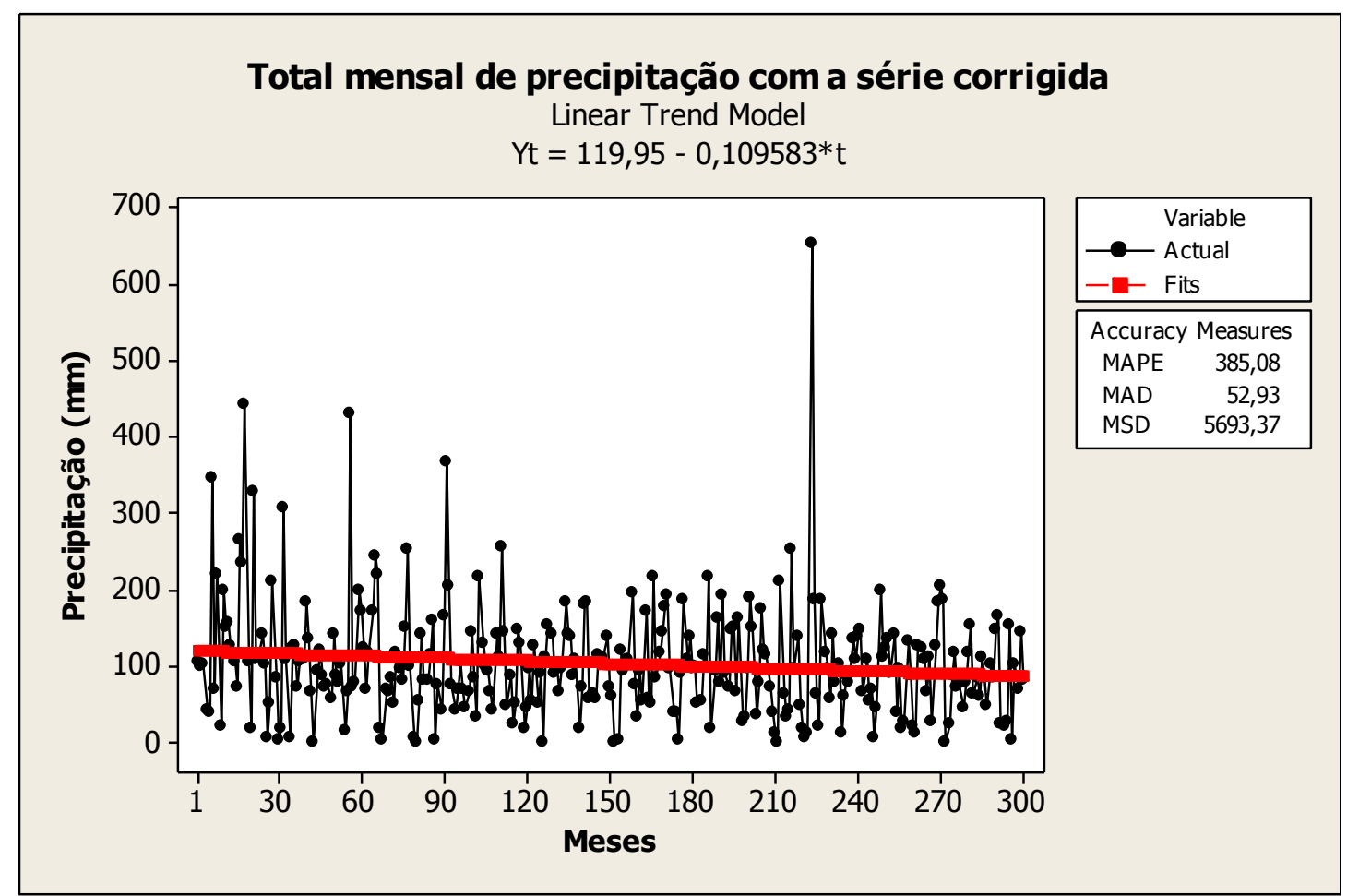

Figura 4 - Total de precipitação mensal da série corrigida do aeroporto de São José dos Campos entre os anos de 1982 a 2006.

Fonte: ICEA. 
Os resultados do teste "Runs" das séries de precipitação e de quantidade de registro também corroboram com a tendência encontrada na análise linear; nos três casos, diminuíram no decorrer da série analisada.

Considerando $\mathrm{K}$ o valor médio de cada série, àquela com os totais de precipitação reais apresentou 125 observações de "Runs" acima de K e 175 abaixo. Para a série com total de registros, ocorreram 133 acima de K e 167 abaixo. Já para a série corrigida de totais de precipitação foram 127 observações de "Runs" acima de K contra 173.

\section{CONCLUSÃO}

Portanto, as análises das tendências da precipitação no Aeroporto de São José dos Campos, baseada nas informações mensais dos totais de precipitação e da quantidade de registros ao longo da série, sugere um decréscimo no total mensal e na frequiência de precipitação, tanto na análise de regressão linear quanto na aplicação do teste de nãoaleatoriedade Runs.

\section{AGRADECIMENTOS}

Agradecemos ao Instituto de Controle do Espaço Aéreo (ICEA) por viabilizar a realização deste trabalho e aos demais companheiros da Subdivisão de Climatologia Aeronáutica do ICEA.

\section{REFERÊNCIAS BIBLIOGRÁGICAS}

Araujo, R.T.; Couto, J.C.; Villaron, M.A.; Pinto, M.L.A.; Corrêa, C.S. Estudo das séries de precipitação e nevoeiro entre os anos de 1975-2005 dos aeroportos internacionais de porto alegre, congonhas utilizando teste estatístico não-paramétrico de aleatoriedade com análise da existência de tendência linear. In: Congresso Brasileiro de Meteorologia (CBMET) XV, 2008, São Paulo. Anais...CD-ROM.

Comando da Aeronáutica. Manual de Auxílio de Rotas Aéreas: ROTAER. Rio de Janeiro: Departamento de Controle do Espaço Aéreo, 2005.

Instituto Brasileiro de Geografia e Estatística. Mapa de Unidades de Relevo do Brasil. Rio de Janeiro: Diretoria de Geociências, 2006. Mapa color., $110 \mathrm{~cm}$ x $90 \mathrm{~cm}$. Escala $1: 5.000 .000$

Mapa de Vegetação do Brasil. Rio de Janeiro: Diretoria de Geociências, 2004. Mapa color., $115 \mathrm{~cm}$ x $90 \mathrm{~cm}$. Escala 1:5.000.000.

Jianqing, F.; Yao, Q. Nonlinear time series: Nonparametric and Parametric Methos. Spring. Verlag. 2003.

Marengo, J.A. ; Alves L.M. T.endências hidrológicas da bacia do Rio Paraíba do Sul. Revista Brasileira de Meteorologia, v.20, n.2, p.215-226, ago. 2005.

Paula, G.R. O eucalipto e o ciclo hidrológico no município de São Luiz do Paraitinga, SP. Monografia. (Graduação em Geografia). Universidade de Taubaté,Taubaté, 2008. 
Salgueiro, J.H.P.B.; Montenegro S.M.G.L. Análise da distribuição espacial da precipitação na bacia do rio Pajeú em Pernambuco segundo o método geoestatístico. Rev. Tecnol. Fortaleza, v. 29, n. 2, p.174-174 185, dez. 2008.

Santos, A. M. dos; Galvíncio, J. D. Moura, M S. B. de. Homogeneização da precipitação pluviométrica na bacia Hidrográfica do Rio Goiana - PE, com método de análise de Agrupamento. RBGF - Revista Brasileira de Geografia Física Recife-PE Vol. 01 n.01 Mai/Ago 2008, 14-27

Sheskin, D.J. Handbook parametrc and nonparametric statistical procedures. Chapman \& Hall/CRC. 2000.

Siegel, S.; Castellan, N. J., Jr.. Nonparametric statistics for the behavioral sciences. $\left(2^{\text {nd }}\right.$ ed.) New york: McGraw-Hill Book Company, 1988.

Villaron, M.A. Análise Temporal das Condições de Nevoeiro no Aeroporto de São José dos Campos entre os anos de 1982 e 2004. 2008. 52p. Monografia. (Graduação em Geografia).Universidade de Taubaté,Taubaté, 2003. 